\title{
Recombination characteristics of therapeutic ion beams on ion chamber dosimetry
}

\author{
Naruhiro Matsufuji*, Tetsuharu Matsuyama ${ }^{\dagger}$ and Shinji Sato ${ }^{\ddagger}$ \\ Medical Physics Research Program, National Institute of Radiological Sciences, 9-1, Anagawa-4, Inage-ku \\ Chiba-shi, Chiba 263-8555, Japan \\ "matufuji@nirs.go.jp, †t-matsu@nirs.go.jp, ${ }^{\dagger}$ shin_s@nirs.go.jp \\ Toshiyuki Kohno \\ Department of Energy Sciences, Tokyo Institute of Technology, 4259 Nagatsuta-cho, Midori-ku \\ Yokohama-shi, Kanagawa 226-8503, Japan \\ kohno@es.titech.ac.jp
}

Published 1 September 2016

\begin{abstract}
In heavy ion radiotherapy, ionization chambers are regarded as a standard for determining the absorbed dose given to patients. In ion dosimetry, it is necessary to correct the radiation quality, which depends on the initial recombination effect. This study reveals for the radiation quality dependence of the initial recombination in air in ion dosimetry. Ionization charge was measured for the beams of protons at 40-160 MeV, carbon at $21-400 \mathrm{MeV} / \mathrm{n}$, and iron at $23.5-500 \mathrm{MeV} / \mathrm{n}$ using two identical parallel-plate ionization chambers placed in series along the beam axis. The downstream chamber was used as a monitor operated with a constant applied voltage, while the other chamber was used for recombination measurement by changing the voltage. The ratio of the ionization charge measured by the two ionization chambers showed a linear relationship with the inverse of the voltage in the high-voltage region. The initial recombination factor was estimated by extrapolating the obtained linear relationship to infinite voltage. The extent of the initial recombination was found to increase with decreasing incident energy or increasing atomic number of the beam. This behavior can be explained with an amorphous track structure model: the increase of ionization density in the core region of the track due to decreasing kinetic energy or increasing atomic number leads to denser initial ion production and results in a higher recombination probability. For therapeutic carbon ion beams, the extent of the initial recombination was not constant but changed by $0.6 \%$ even in the target region. This tendency was quantitatively well reproduced with the track-structure based on the initial recombination model; however, the transitional change in the track structure is considered to play an important role in further understanding of the characteristics of the initial recombination.
\end{abstract}

Keywords: initial recombination; track structure; ionization chamber.

PACS numbers: 87.53.Bn, 34.80.Lx, 87.53.-j.

\section{Introduction}

In radiotherapy, ionization chambers are regarded as a standard reference tool for measuring the absorbed dose given to patients. The protocol for the ionization chamber dosimetry is specified by, for example, the International Atomic Energy Agency in the

This is an Open Access article published by World Scientific Publishing Company. It is distributed under the terms of the Creative Commons Attribution 3.0 (CC-BY) License. Further distribution of this work is permitted, provided the original work is properly cited. 
TRS-398 protocol for therapeutic radiation treatments. ${ }^{1}$ The principle of the ionization chamber dosimetry can be expressed as

$$
D=\frac{Q_{\infty}}{e} \frac{\mathrm{W}}{\mathrm{m}}=\frac{Q_{V}}{e} \frac{\mathrm{W}}{\mathrm{m}} K_{s} .
$$

Here, $D, W, m$, and $e$ are the absorbed dose, the average energy required to create an ionization pair in the medium gas (called the $W$ value), the mass of the gas, and the elementary charge, respectively. $Q_{\infty}$ represents the amount of positive or negative charge initially produced in the medium gas. The generated ions reach the electrode of the ionization chamber, and some may recombine with the ions of opposite charge. If recombination occurs, the measured charge under voltage $V, Q_{V}$, is smaller than $Q_{\infty}$, leading to underestimation of the absorbed dose. The recombination correction factor $K_{s}$ is therefore defined as the ratio of the amount of the charge initially produced $\left(Q_{\infty}\right)$ and that actually collected $\left(Q_{V}\right)$, such that $K_{s}=Q_{\infty} / Q_{V}$.

While X-rays are currently the most widely used type of radiation for cancer treatment, there is a growing interest in the use of swift ions for therapeutic purposes. ${ }^{2}$ Due to the physical effect known as the Bragg curve, energy loss of incident ions increases gradually in the patient's body until the ions stop. The greater energy loss toward the end of the range enables localized dose delivery to deep-seated tumors. Furthermore, in the case of light ions such as carbon, the Bragg curve is more enhanced in biological systems due to the increasing biological effectiveness associated with the increasing energy density. These characteristics of carbon ions have been shown to enable effective treatment of tumors that are radioresistant to X-rays and to realize hypofractionated radiotherapy without causing severe side effects to the surrounding normal tissues. ${ }^{3}$

The effect of recombination relevant for ionization chamber dosimetry is considered to be more severe on the ion beams than on the X-rays because of the denser energy loss and denser charge formation by the ions. However, studies on the recombination for ion beams are scarce. ${ }^{4}$ Estimation of quantitatively reliable $K_{s}$ values and the mechanism for recombination are still open questions. Therefore, this study aims to experimentally investigate the recombination characteristics in the ionization chamber for various ion species with various energies as well as to physically model the recombination characteristics.

\section{Materials and Methods}

\subsection{Estimation of the initial recombination correction factor}

The recombination of the produced charge in a dosimeter is categorized in two modes. General (or volume) recombination is an intertrack recombination mode between the positive ions and electrons originating from different radiation incidences, while the initial (or columnar) recombination is an intratrack recombination mode within a single radiation passage. The former is significant only under a high dose rate field and is considered to be negligible in therapeutic applications. 
According to Boag theory, ${ }^{5}$ the charge collection efficiency $f$ and its inverse, i.e. the initial recombination correction factor $K_{s}$, are given by the ratio of the amount of charge initially produced $\left(Q_{\infty}\right)$ to that actually collected $\left(Q_{V}\right)$ as

$$
f=\frac{1}{K_{S}}=\frac{Q_{V}}{Q_{\infty}}=\frac{1}{u} \ln (1+u) .
$$

For a parallel-plate ionization chamber, $u$ is given as a function of the supplied voltage $V$ and the gap distance $d$ with a gas-dependent coefficient $\mu$ as

$$
u=\mu \frac{Q_{\infty} d^{2}}{V} .
$$

For a pulsed beam, Eq. (2) is simplified as

$$
\frac{1}{f}=K_{s}=1+u=1+C \frac{Q_{\infty}}{V}=\frac{Q_{\infty}}{Q_{V}} .
$$

This suggests that a linear relationship can be expected between the inverse of the supplied voltage $1 / V$ and that of the corrected charge $1 / Q_{v}$ :

$$
\frac{1}{Q_{V}}=\frac{1}{Q_{\infty}}+C \frac{1}{V}
$$

The initial charge $Q_{\infty}$ can be derived as an intercept of the $1 / Q_{v}$ axis when a linear fit is applied to the $1 / Q_{v}$ vs. $1 / V$ plot obtained from the measurements.

\subsection{Experiment}

\subsubsection{Site}

Experiments were conducted at the Secondary Beam Line 2 (SB2) of the Heavy Ion Medical Accelerator in Chiba (HIMAC) with beams of ${ }^{1} \mathrm{H}(40-60 \mathrm{MeV}),{ }^{4} \mathrm{He}(24-230$ $\mathrm{MeV}),{ }^{12} \mathrm{C}(21-400 \mathrm{MeV} / \mathrm{n})$, and ${ }^{56} \mathrm{Fe}(24-500 \mathrm{MeV} / \mathrm{n})$. To change the incident beam energy, the SB2 line is equipped with an energy degrader consisting of different thicknesses of beryllium plates. Mass analyzing magnets are also provided downstream of the energy degrader to select the energy spreading and eliminate fragment particles.

\subsubsection{Setup}

To experimentally derive the initial recombination correction factor, two PTW 34014 parallel-plate ionization chambers were used. The chambers were carefully positioned in series at the center of the beam line and perpendicular to the beam axis. The chamber has a large sensitive volume $\left(86 \mathrm{~cm}^{3}, 148 \mathrm{~mm}\right.$ in diameter and $2.5 \mathrm{~mm}$ in thickness) to cover the entire radiation track. The sensitive volume was vented to air under atmospheric pressure. The upstream chamber was used to investigate the recombination characteristics $\left(Q_{V}\right)$ by changing the supplied voltage, while the downstream chamber was used as a monitor $\left(Q_{m}\right)$ with a constant voltage of $500 \mathrm{~V}$. $K_{s}$ can then be experimentally derived by normalizing $Q_{\infty}$ and $Q_{V}$ by $Q_{m}$ according to Eq. (6): 


$$
K_{s}=\frac{Q_{\infty}}{Q_{V}}=\frac{Q_{\infty} / Q_{m}}{Q_{V} / Q_{m}}
$$

The incident beam was focused to be about $1 \mathrm{~cm}$ in diameter at the entrance window. The charges produced in the chambers were monitored by Keithley 6514 electrometers for changing supplied voltages provided by Oxford TC952A HV DC Supply from 300 to $500 \mathrm{~V}$ in $50 \mathrm{~V}$ steps for each beam. The $Q_{\infty} / Q_{m}$ value was estimated using a linear fit to the four obtained data points.

\subsection{Modeling of initial recombination}

\subsubsection{Track structure}

Although energy deposition along a single radiation track is a highly random event, an amorphous dose distribution along the track can be estimated using the statistically expected form. Kiefer and Chatterjee $(\mathrm{K}-\mathrm{C})$ proposed that for the radial track structure created by an incident ion with energy $E$, velocity $v$, and atomic number $A$, the track shape can be characterized using the $r_{c}$ and $r_{p}$ parameters given by ${ }^{6-7}$

$$
\begin{gathered}
r_{c}=0.116 \times \frac{v}{c}, \text { and } \\
r_{p}=0.0616 \times\left(\frac{E}{A}\right)^{1.7},
\end{gathered}
$$

where $c$ is the speed of light. $r_{c}$ corresponds to the maximum radial distance of the "core" region from the track center where the incident ion directly ionizes the medium, while $r_{p}$ specifies the maximum distance of the "penumbra" region where energy deposition is caused by secondary electrons ejected from the core region.

Using these parameters, the radial energy distribution $D(r)$ is given by

$$
\begin{gathered}
D_{c}(r)=\frac{1}{\pi r_{c}^{2}}\left[\frac{L E T}{\rho_{\text {air }}}-2 \pi K_{p} \log \left(\frac{r_{p}}{r_{c}}\right)\right], \text { and } \\
D_{p}(\mathrm{r})=K_{p} \times r^{-2},
\end{gathered}
$$

with

$$
K_{p}=1.25 \times 10^{-4}\left(z^{*} / \beta\right)^{2} .
$$

Here, $z^{*}, \beta$, and $\rho_{\text {air }}$ are the ion's effective charge, speed relative to that of light, and density of air, respectively.

Assuming that the number of initial ion pairs at a point $r, n(r)$, is proportional to the local dose $D(r)$ according to $n(r)=\left(\rho_{\text {air }} / W_{\text {air }}\right) D(r)$, the extent of recombination $\Delta Q$ can be estimated as

$$
\Delta Q=\mathrm{eC}_{R} \frac{d}{V} \int_{0}^{r_{p}} n(r)^{2} 2 \pi r d r=\mathrm{eC}_{R} \frac{d}{V}\left[\pi n_{c} r_{c}^{2}+\int_{r_{c}}^{r_{p}} n(r)^{2} 2 \pi r d r\right]
$$

where $C_{R}$ is the proportionality coefficient for the recombination. On the other hand, from Eq. (1), $Q_{\infty}$ is given here as 


$$
Q_{\infty}=\mathrm{e} \frac{L E T}{W_{\text {air }}}
$$

Then,

$$
\frac{\Delta Q}{Q_{\infty}}=\mathrm{eC}_{R} \frac{d}{V} \frac{W_{a i r}}{L E T}\left[\pi n_{c} r_{c}^{2}+\int_{r_{c}}^{r_{p}} n(r)^{2} 2 \pi r d r\right] \equiv \mathrm{C}_{R} \cdot \Delta q
$$

The experimentally derived $K_{s}$ can then be rewritten as

$$
K_{S}=\frac{Q_{\infty}}{Q_{V}}=\frac{1}{1-\frac{\Delta Q}{Q_{\infty}}}=\frac{1}{1-C_{R} \cdot \Delta q} .
$$

$\Delta \mathrm{q}$ is derived from calculation, and $\mathrm{C}_{\mathrm{R}}$ is an adjustable parameter for bridging the experiment and the calculation, i.e. $K_{s}$ and $\Delta q$.

\section{Results}

Figure 1 shows the experimental results for the recombination correction factor $K_{s}$ as a function of incident energy for the various ion beams. The extent of the initial recombination is not constant but changes depending on the radiation quality. $K_{s}$ becomes more significant when the deposited energy density LET increases, i.e. for the incidence of slower and heavier ions. In the case of the Fe beam, initial recombination of more than $10 \%$ was observed.

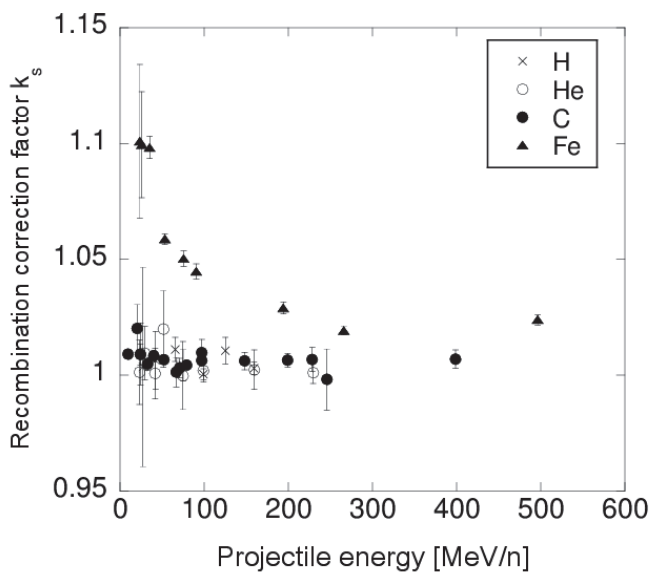

Fig. 1. Experimental results for the initial recombination correction factor $K_{s}$ as a function of incident energy.

\section{Discussion}

\subsection{Parameters of the initial recombination model}

Figure 2 shows the $\Delta q$ values for the ion beams used in the experiment, calculated using Eqs. (7-11). The $C_{R}$ proportionality parameter values shown were determined in order to 
reproduce the experimental recombination results (Fig. 1), with the extent of recombination estimated using the $\mathrm{K}-\mathrm{C}$ track structure (Fig. 2) according to Eq. (15). The obtained results are shown in Fig. 3 as a function of $\Delta q$.

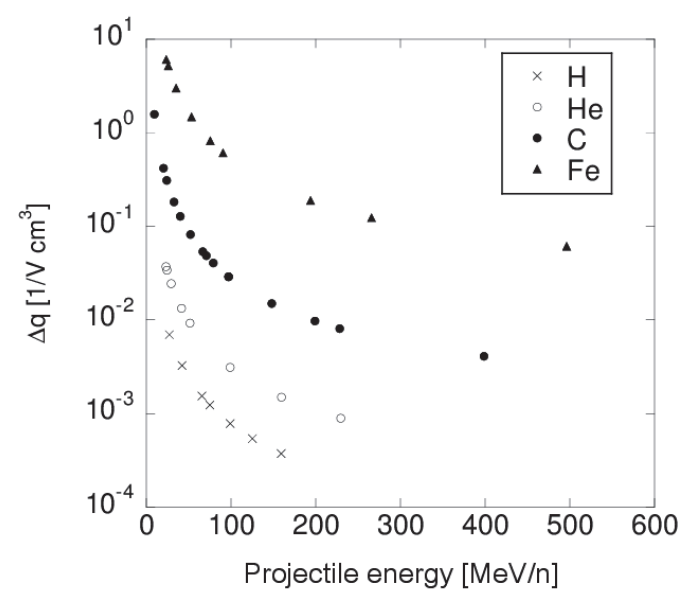

Fig. 2. Extent of recombination $\Delta q$ calculated with the $\mathrm{K}-\mathrm{C}$ track structure model for beams used in this study.

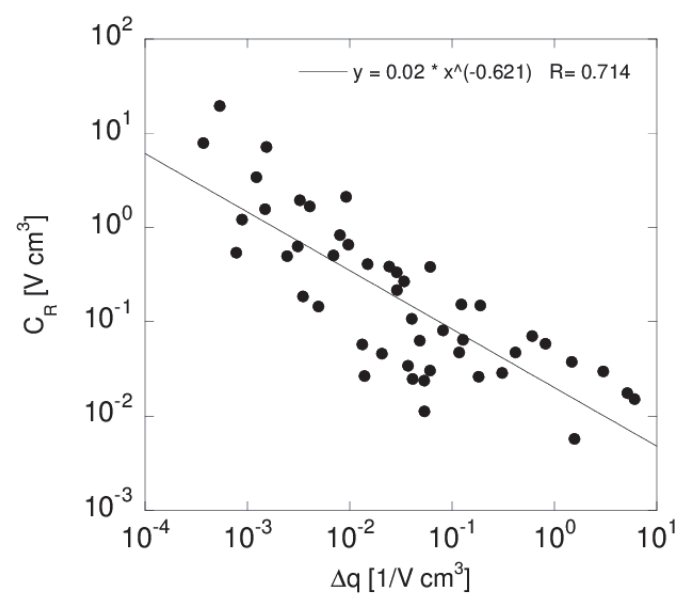

Fig. 3. Dependence of proportionality factor $C_{R}$ on $\Delta q$.

As shown in Fig. $3, C_{R}$ is inversely proportional to $\Delta q$. By fitting the obtained $C_{R}$ values to a power function of $\Delta q$, the following relationship is derived:

$$
C_{R}=0.02 \times \Delta q^{-0.621} \text {. }
$$


Finally, by combining Eqs. (15) and (16), the following relationship is derived to specify the initial recombination of the ion beams:

$$
K_{S}=\frac{1}{1-C_{R} \cdot \Delta q}=\frac{1}{1-0.02 \cdot \Delta q^{0.379}} .
$$

\subsection{Estimation of initial recombination of therapeutic carbon ion beam}

We now estimate the extent of initial recombination of a therapeutic carbon ion beam based on the modeled initial recombination characteristics derived above. As mentioned in the introduction, ionization density of incident ions increases toward the end of range. To deliver a uniform dose to the entire target, the pristine Bragg curve is appropriately superimposed to shape the so-called spread-out Bragg peak (SOBP) beam. ${ }^{8}$ In addition, in the case of carbon ion radiotherapy, some fragment particles generated by nuclear reactions between the incident carbon ions and the target element contaminate the beam. Therefore, the therapeutic carbon ion beam exhibits a complex radiation quality due to the presence of various ion species of different energies. To estimate the influence of the initial recombination on the dosimetry of the therapeutic carbon ion beam, the elemental energy spectra as a radiation quality $\Phi(E, Z)$ in water were first simulated using the PHITS $^{9}$ Monte-Carlo simulation code for the commonly used $400 \mathrm{MeV} / \mathrm{n}$ carbon beam at the proximal $(183 \mathrm{~mm})$, middle $(213 \mathrm{~mm})$, and distal end $(243 \mathrm{~mm})$ within $60 \mathrm{~mm}$ SOBP. As an example, the simulated $\Phi(E, Z)$ at the middle of $60 \mathrm{~mm}$ SOBP is shown in Fig. 4.

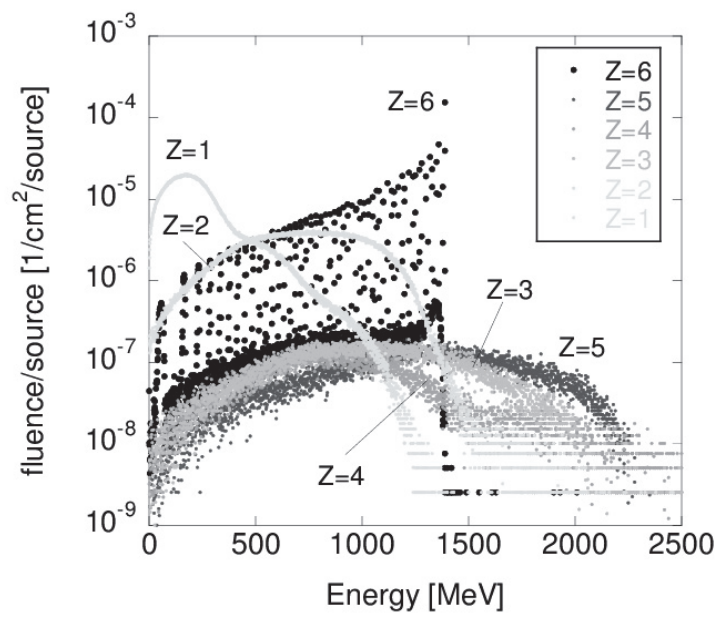

Fig. 4. Elemental energy spectra at the middle of the $60 \mathrm{~mm} \mathrm{SOBP}$ of C-400 MeV/n beam obtained by simulations using PHITS.

The total extent of the initial recombination $\Delta Q_{\text {total }}$ was estimated as

$$
\Delta Q_{\text {total }}=\sum_{Z=1}^{6} \int_{0}^{\infty} \Phi(E, Z) \Delta Q(E, z) d E,
$$


where

$$
\Delta Q(E, Z)=e^{\frac{L E T_{\infty}(E, Z)}{W_{\text {air }}}} C_{R}(E, Z) \Delta q(E, z),
$$

and the results are shown in Fig. 5 together with the experimental data.

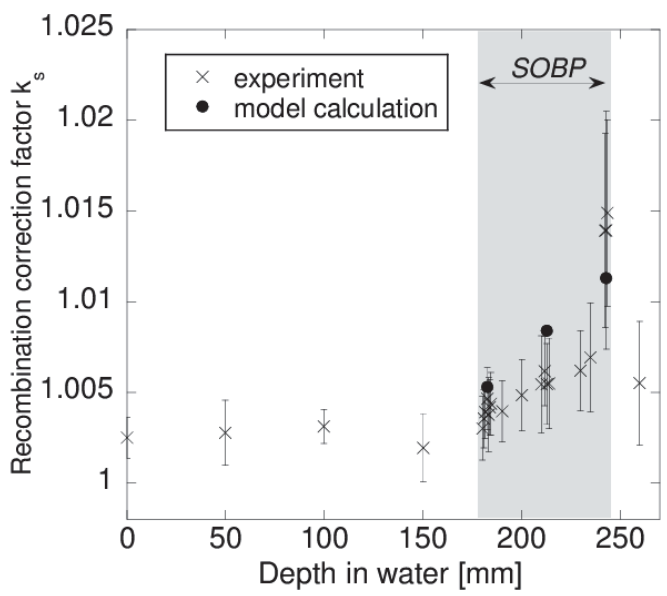

Fig. 5. Extent of the initial recombination of therapeutic $400 \mathrm{MeV} / \mathrm{n} \mathrm{C}$. Crossed symbols are experimental data, and closed circles are data obtained by model calculations. Shaded area corresponds to the SOBP region.

As shown in Fig. 5, the recombination model derived by this study is applicable even for the quantitative estimation of recombination extent within the experimental error for the complex therapeutic carbon ion beam. Even within the SOBP target region, the extent of recombination gradually increases toward the distal end by about $0.6 \%(0.5 \%-1.1 \%)$. Because the extent of variation is comparable with the relative dosimetry precision in routine quality assurance (QA), consideration of the initial recombination as well as its dependence on the radiation quality will contribute to further optimization of carbon ion radiotherapy in the future.

\subsection{Consideration of the track structure for ion recombination}

As appeared in Fig. 3, the decrease in $C_{R}$ means that the extent of the initial recombination is less significant than expected with the $\mathrm{K}-\mathrm{C}$ track structure model for densely ionizing radiation with higher $\Delta q$. As one of the possible reasons, this tendency can be attributed to the kinetic diffusion process of secondary electrons from the core: as previously explained, all the secondary electrons are initially produced in the core and then gradually spread to the penumbra. The $\mathrm{K}-\mathrm{C}$ track structure model corresponds to the fully distributed final stage; however, the initial recombination process can take place even at the earlier distributing stage. To consider the influence, the initial recombination is revaluated with Eq. (14) but initial track structure made of core only. There the radius of the core is fixed to $10 \mathrm{~nm}$ as introduced in the Local Effect Model, ${ }^{10}$ which has been 
utilized as a biological model in a treatment-planning system of carbon ion radiotherapy in Germany. The resultant recombination coefficient $C_{R}$ is shown for carbon and iron beams as Fig. 6.

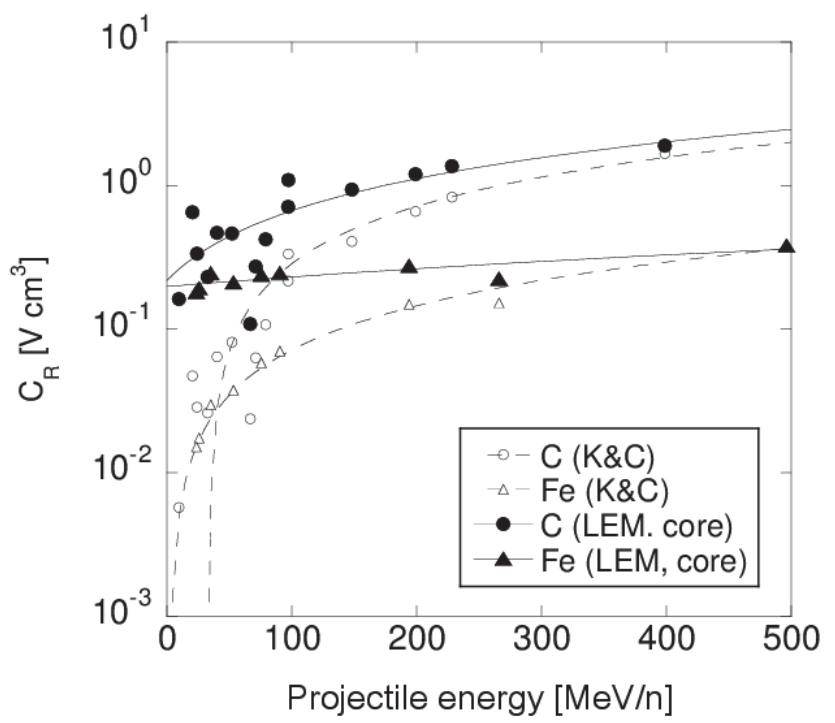

Fig. 6. Change in the initial recombination coefficient $C_{R}$ for carbon and iron beams as a function of incident energy. Closed symbols are obtained by the core portion of the track structure used in LEM and open symbols are obtained by $\mathrm{K}-\mathrm{C}$ track structure. Lines in the figure correspond to linear fit to the symbols for eye guide.

As shown in the figure, the drastic decrease in $C_{R}$ for less energetic, i.e. higher $\Delta q$, ions found on $\mathrm{K}-\mathrm{C}$ track model estimation is significantly improved by taking only LEM-based core portion into account. On the other hand, $C_{R}$ is not unique but still ion species dependent between carbon and iron beams. In a future study, the continuous transitional change from the initial core to the final core-penumbra will be considered in the initial recombination model in order to understand the mechanism.

\section{Conclusion}

The initial recombination characteristics of ionization chamber dosimetry for swift ion beams were studied for a therapeutic carbon ion beam as well as for proton and iron beams. Over $10 \%$ of the initial ions are lost by recombination for the low-energy iron beam. In the case of therapeutic carbon ions, the extent of the initial recombination is about $1 \%$; however, the ratio is not constant but varies by about $0.6 \%$ even in the SOBP region. In contrast to the routine QA precision, this variation is not negligible. The characteristics of initial recombination are well reproduced by taking into account the radial track structure formed by each ion. It is suggested that the transitional change in 
the track structure plays an important role in understanding the characteristics of the initial recombination.

\section{Acknowledgments}

The authors are thankful for the skillful work of HIMAC operators. A part of this study was carried out as a Research Project with Heavy Ions at NIRS-HIMAC (14H332) and composed of a master thesis of Matsuyama. ${ }^{11}$

\section{References}

1. IAEA, Absorbed Dose Determination in External Beam Radiotherapy, Technical Report Series No. 398 (IAEA, Vienna, 2000).

2. M. Jermann, Int. J. Particle Therapy 1, 40 (2014).

3. H. Tsujii and T. Kamada, Jpn. J. Clin. Oncol. 42, 670 (2012).

4. T. Kanai et al., Phys. Med. Biol. 43, 3549 (1998).

5. J. W. Boag and J. Currant, Br. J. Radiol. 53, 471 (1980).

6. J. Kiefer and H. Straaten, Phys. Med. Biol. 31, 1201 (1986).

7. A. Chatterjee and H. J. Schaefer, Radiat. Environm. Biophys. 13, 215 (1976).

8. T. Kanai et al., Int. J. Radiation Oncol. Biol. Phys. 44, 201 (1999).

9. T. Sato et al., J. Nucl. Sci. Technol. 50, 913 (2013).

10. M. Scholz and G. Kraft, Adv. Space Res. 18, 5 (1996).

11. T. Matsuyama, master thesis of the Graduated School of Toyo Institute of Technology (in Japanese) (2015). 